\title{
In-situ electron microscopy study of non-volatile resistive switching in Mott insulator $\mathrm{VO}_{2}$
}

Shaobo Cheng ${ }^{1}$, Min-Han Lee ${ }^{2}$, Xing $\mathrm{Li}^{3}$, Lorenzo Fratino ${ }^{4}$, Marcelo Rozenberg ${ }^{4}$, Ivan Schuller ${ }^{5}$ and Yimei Zhu 6

${ }^{1}$ Department of Condensed Matter Physics and Materials Science, Brookhaven National Laboratory, Upton, NY, USA, upton, New York, United States, ${ }^{2}$ University of California-San Diego, United States,

${ }^{3}$ Key Laboratory of Material Physics, School of Physics and Microelectronics, Zhengzhou University, Zhengzhou, Henan, P. R. China, United States, ${ }^{4}$ Laboratoire de Physique des Solides, CNRS, Université Paris-Sud, Université Paris-Saclay, Orsay, France, United States, ${ }^{5}$ Materials Science and Engineering Program, University of California-San Diego, La Jolla, CA, USA, United States, ${ }^{6}$ Department of Condensed Matter Physics and Materials Science, Brookhaven National Laboratory, Upton, NY, USA, United States

In-situ transmission electron microscopy (TEM) has been widely used for resolving the nanoscale changes (both structurally and electronically) on the functional devices. [1-3] $\mathrm{VO}_{2}$, a typical Mott insulator, has near room temperature metal-insulator-transition (MIT), which has great potential in the applications for electronic devices. [4,5] The MIT is the result of the rutile-monoclinic structure transition and features volatile resistive switching. It is intuitive to wonder whether the nonvolatile resistive switching behavior can be triggered within the same system as well.

In this work, both ex-situ device measurements and in-situ biasing TEM studies have been combined to show the nonvolatile switching and recovery behaviors in $\mathrm{VO}_{2}$ based device.[6] As shown in Fig.1, a 150 $\mathrm{nm} \mathrm{VO}_{2}$ thin film is sandwiched by Au top electrodes and a Ga-doped Ge conductive bottom electrode, whose configuration will benefit the future 3D crossbar-shape circuits. By using the ex-situ device measurements, different resistive switching behaviors can be realized under different temperatures. The volatile switching occurs near the phase transition temperature, while the non-volatile one is realized at lower temperature.

In-situ biasing TEM experimental results reveal the fact that the applied bias can not only induces the phase transition via Joule heating, but also triggers the electroforming process. The competition between Joule heating and electroforming process varies with the temperature. As shown in Fig. 2, a new metallic Magnéli phase $\mathrm{V}_{5} \mathrm{O}_{9}$ has been created by electroforming process and forms new conductive filament. The reversibility of this conductive filament was also verified by annealing the sample in oxygen environment, which helps to (partially) relax the $\mathrm{V}_{5} \mathrm{O}_{9}$ conductive filament. The change of transportation property was simultaneously measured and results are shown in Fig. 2(f). With partially relaxed conductive filament, a smaller voltage is needed to recreate the $\mathrm{V}_{5} \mathrm{O}_{9}$ conductive pathway in the second measurement. It is worthwhile to mention that, unlike the $\mathrm{TiO}_{2}$ system, [3] the reset process of non-volatile resistive switching cannot be realized in $\mathrm{VO}_{2}$ system due to the fact monoclinic-to-rutile phase transition will be triggered under large electric current. 
In conclusion, we identify, for the first time, the structure (Magnéli phase $\mathrm{V}_{5} \mathrm{O} 9$ ) of the conductive filament during the electroforming process using in-situ microscopy and the mechanism of the non-volatile switching in $\mathrm{VO}_{2}$. We have demonstrated that two different resistive switching behaviors can be realized using the $\mathrm{VO}_{2} / \mathrm{Ge}$ resistive switching device, which is of great interest to emulate both neuronal and synaptic behaviors for the future neuromorphic computing on the same system.

(a)
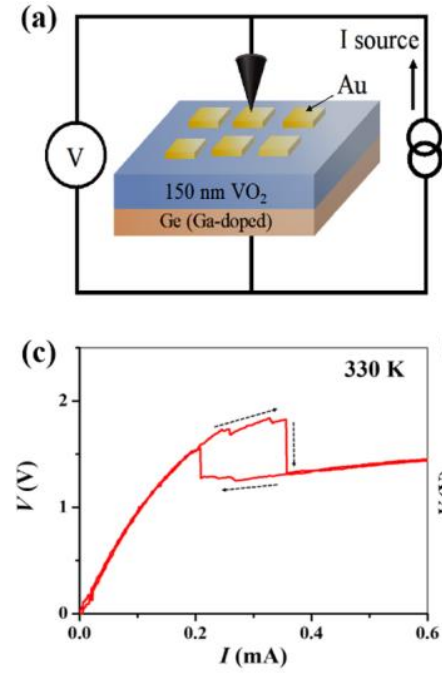
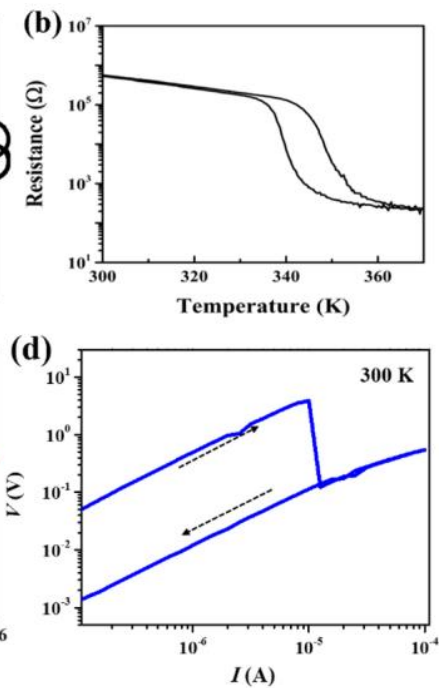

Figure 1. Ex-situ device measurement for the $\mathrm{Au} / \mathrm{VO} 2 / \mathrm{Ge}$ system. (a) The out-of-plane device configuration for transportation measurement. Au patterns are deposited and used as top electrode. (b) Resistance vs temperature curve showing near four order change in resistance across the metal-insulator transition on pristine VO2 sample. (c) The typical volatile resistive switching behavior measured at 330 $\mathrm{K}$. (d) Measured nonvolatile resistive switching behavior for the same device under $300 \mathrm{~K}$.
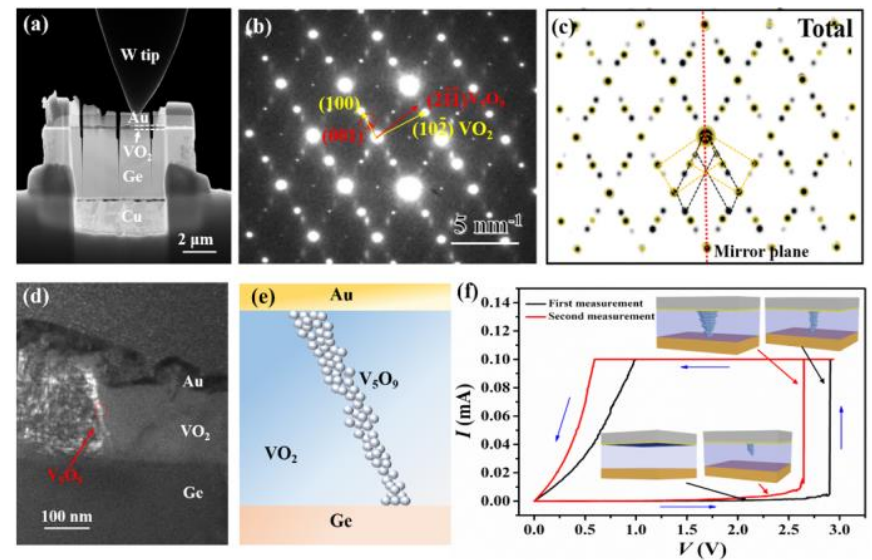

Figure 2. In situ biasing TEM studies on $\mathrm{Au} / \mathrm{VO} 2 / \mathrm{Ge}$ out-of-plane device at room temperature. (a) Low magnification HAADF-STEM image showing the experimental setup. (b) Selected area electron diffraction pattern acquired after applying bias. Besides the diffraction spots from VO2, the V5O9 phase can be identified. (c) The simulated electron diffraction pattern of combined VO2 \& V5O9 phases. (d) Dark field TEM image showing the formation of V5O9 phase conductive filament after applying biasing using the V5O9 (001) diffraction spot. (e) Schematic model of the V5O9 filament. (f) The simultaneously acquired I-V curves in biasing TEM experiments. The second measurement was conducted after partially 
removing the Magnéli phase V5O9 in Oxygen. The embedded schematic diagrams showing the evolution of the conductive filament.

References

[1] Sun, W. et al., Nature Communications 10 (2019) 3453

[2] Cheng S., et al., Advanced Electronic Materials, 5 (2019) 1800827

[3] Kwon, D. -H. et al. Nature Nanotechnology 5 (2010) 148-153

[4] Valle, J. D., Ramírez, J. G., Rozenberg, M. J. \& Schuller, I. K., Journal of Applied Physics. 124 (2018) 211101

[5] Valle, J. D. et al., Nature, 569 (2019) 388-392

[6] Cheng S., et al., Proceedings of the National Academy of Sciences of the United States of America, 118 (2021) e2013676118

[7] This work is supported by the Quantum Materials for Energy Efficient Neuromorphic Computing, an Energy Frontier Research Center funded by the U.S. Department of Energy (DOE), Office of Science, Basic Energy Sciences (BES), under Grant No. DE-SC0019273. Electron microscopy work at BNL was supported by the U.S. Department of Energy, Office of Basic Energy Science, Division of Materials Science and Engineering, under Contract No. DE-SC0012704. 